\title{
General anesthesia with remimazolam in a patient with mitochondrial encephalomyopathy: a case report
}

\author{
Yuji Suzuki* ${ }^{*}$, Matsuyuki Doi and Yoshiki Nakajima
}

\begin{abstract}
Background: Systemic anesthetic management of patients with mitochondrial disease requires careful preoperative preparation to administer adequate anesthesia and address potential disease-related complications. The appropriate general anesthetic agents to use in these patients remain controversial.

Case presentation: A 54-year-old woman (height, $145 \mathrm{~cm}$; weight, $43 \mathrm{~kg}$ ) diagnosed with mitochondrial encephalomyopathy with lactic acidosis and stroke-like episodes underwent elective cochlear implantation. Infusions of intravenous remimazolam and remifentanil guided by patient state index monitoring were used for anesthesia induction and maintenance. Neither lactic acidosis nor prolonged muscle relaxation occurred in the perioperative period. At the end of surgery, flumazenil was administered to antagonize sedation, which rapidly resulted in consciousness.
\end{abstract}

Conclusions: Remimazolam administration and reversal with flumazenil were successfully used for general anesthesia in a patient with mitochondrial disease.

Keywords: Remimazolam, Mitochondrial encephalomyopathy with lactic acidosis and stroke-like episodes, Mitochondrial myopathy, Flumazenil

\section{Background}

Mitochondrial diseases are caused by abnormalities in mitochondrial or nuclear genes that result in abnormal mitochondrial morphology or function $[1,2]$. Cellular energy production is therefore disordered and organs with high energy demand are most affected [2]. Disease symptoms range from mild to severe and may involve multiple organs. Examples include ataxia, hearing loss, convulsions, cognitive decline, cardiomyopathy, myocardial conduction disorders, limb weakness, ophthalmoplegia, and diabetes $[1,3]$. Systemic anesthetic management of patients with mitochondrial disease requires careful preoperative preparation to administer adequate anesthesia and address potential disease-related complications. However, clinical

\footnotetext{
* Correspondence: alutena@gmail.com

Department of Anesthesiology and Intensive Care Medicine, Hamamatsu University School of Medicine, 1-20-1 Handayama, Hamamatsu, Shizuoka 431-3192, Japan
}

\section{Springer Open}

trials of anesthetic agents in adult mitochondrial myopathy patients have not been performed. The appropriate agents to use remain controversial [4].

Recently, remimazolam, an ultrashort-acting benzodiazepine, was approved for clinical use in Japan and is now available. Remimazolam is rapidly degraded by carboxylesterases in the liver and its metabolites have negligible pharmacological activity [5]. We report the successful use of intravenous remimazolam and its antagonist flumazenil for general anesthesia in a patient with mitochondrial myopathy.

\section{Case presentation}

A 54-year-old woman (height, $145 \mathrm{~cm}$; weight, $43 \mathrm{~kg}$ ) diagnosed with mitochondrial encephalomyopathy with lactic acidosis and stroke-like episodes elected to undergo cochlear implantation to address her progressive bilateral sensorineural deafness. Preoperative electrocardiography (c) The Author(s). 2021 Open Access This article is licensed under a Creative Commons Attribution 4.0 International License, which permits use, sharing, adaptation, distribution and reproduction in any medium or format, as long as you give appropriate credit to the original author(s) and the source, provide a link to the Creative Commons licence, and indicate if changes were made. The images or other third party material in this article are included in the article's Creative Commons licence, unless indicated otherwise in a credit line to the material. If material is not included in the article's Creative Commons licence and your intended use is not permitted by statutory regulation or exceeds the permitted use, you will need to obtain permission directly from the copyright holder. To view a copy of this licence, visit http://creativecommons.org/licenses/by/4.0/. 
showed no abnormalities. Transthoracic echocardiography showed circumferential thickening of the left ventricle and asymmetric hypertrophy of the septum, which was thicker than the posterior wall. Cardiac contractility was diffusely decreased and cardiac ejection fraction was $45 \%$. Estimated glomerular filtration rate was low $(27 \mathrm{~mL} / \mathrm{min})$ because of diabetic nephropathy. Although enhanced insulin therapy had been introduced, her HbA1c level was high (12.5\%) immediately before surgery. Creatine kinase level was $162 \mathrm{IU} / \mathrm{L}$ (normal range, 41-153). Her medications included $5 \mathrm{mg}$ oral imidapril daily and subcutaneous insulin (6 units in the morning, 8 units in the afternoon, and 6 units in the evening). She was not taking any antiepileptic drugs.

No premedication was administered. Intravenous remimazolam was administered as a $0.2 \mathrm{mg} / \mathrm{kg}$ bolus over $1 \mathrm{~min}$, which resulted in loss of consciousness, followed by continuous infusion of remimazolam $(1 \mathrm{mg} /$ $\mathrm{kg} / \mathrm{h})$ and remifentanil $(0.2 \mu \mathrm{g} / \mathrm{kg} / \mathrm{min})$. Neuromuscular monitoring of the left ulnar nerve was initiated using a train-of-four (TOF) stimulus (TOF watch SX, MSD, Japan). Three minutes after administration of $30 \mathrm{mg}$ of intravenous rocuronium, all four twitch responses disappeared and tracheal intubation was performed. During surgery, remimazolam was administered along with a continuous remifentanil infusion $(0.2-0.25 \mu \mathrm{g} / \mathrm{kg} / \mathrm{min})$ to maintain the patient state index value between 25 and 50. Patient state index was measured using the SEDLine ${ }^{\circ}$ monitor (Masimo Inc., Irvine, CA). A catheter was placed in the right radial artery for continuous arterial pressure monitoring. Intermittent blood gas analysis showed that the lactate concentration and $\mathrm{pH}$ ranged from 1.8 to $1.9 \mathrm{mmol} / \mathrm{L}$ and 7.41 to 7.45 , respectively. Surgical time was $1 \mathrm{~h}$ and $34 \mathrm{~min}$. Additional rocuronium administration was not needed throughout the surgery. After surgery was completed, the infusions were stopped and the TOF ratio was 0.91 . The patient was extubated after stable spontaneous respirations with tidal volumes $\geq 8 \mathrm{~mL} / \mathrm{kg}$ were confirmed ( $22 \mathrm{~min}$ after the end of surgery and $8 \mathrm{~min}$ after discontinuation of remimazolam). Thirteen minutes after extubation, her eyes remained closed; therefore, $200 \mu \mathrm{g}$ of intravenous flumazenil was administered. Two minutes later, she opened her eyes, became verbally responsive, and was discharged from the operating room. After surgery, her only complaints were sore throat and nausea, which were treated with $1000 \mathrm{mg}$ of acetaminophen and $10 \mathrm{mg}$ of metoclopramide.

\section{Discussion}

Selection of general anesthesia method is a concern in patients with mitochondrial disease [1]. Although the risk of malignant hyperthermia from inhalational anesthetics was previously believed to be increased in patients with muscular disease, this does not appear to be the case, even for patients with hereditary muscular disease. Malignant hyperthermia risk is not higher in patients with muscular dystrophy compared with the general population [6], and the same is assumed for patients with mitochondrial disease [7]. In fact, a survey of pediatric anesthesiologists in the USA showed that approximately $80 \%$ used sevoflurane to induce and maintain anesthesia in children with mitochondrial disease [7]. Propofol infusion syndrome is another concern because its risk is reportedly high in patients with mitochondrial disease [8]. Opinions vary regarding the selection of inhalational anesthetics or propofol. In our patient, we selected a benzodiazepine to avoid the risks of both. Benzodiazepines have been considered safe for general anesthesia in patients with mitochondrial disease and can be administered intravenously [7]. However, clearance is slow and prolonged effects may occur [9].

Remimazolam is an ultrashort-acting benzodiazepine with an imidazobenzodiazepine structure that exhibits pharmacological effects similar to those of midazolam. It has a side chain with an ester bond that is rapidly inactivated by carboxylesterase hydrolysis in the liver. The pharmacological activity of remimazolam metabolites is low, approximately $1 / 400$ th that of remimazolam, which suggests they have negligible pharmacological activity [5]. Furthermore, the effects of remimazolam can be reversed by flumazenil. These features make remimazolam a safe and feasible alternative to inhalational anesthetics and propofol.

The use of muscle relaxants in patients with mitochondrial disease presents potential difficulties. Patients with mitochondrial disease may experience muscle weakness and prolonged muscle relaxation after anesthesia. Muscle relaxant effects may be prolonged in these patients because of increased rocuronium sensitivity $[10,11]$. To address this, sugammadex administration to reverse neuromuscular blockade may be useful, but experience is limited and no clear conclusion has yet been reached. In our patient, we did not administer sugammadex because the TOF ratio was $\geq 0.9$ [12] and the effects of sugammadex in patients with muscular disease and TOF ratio $\geq 0.9$ have not been investigated. Furthermore, sugammadex administration is associated with risks such as difficulty with reintubation and allergic reaction [13]. Therefore, the decision to use sugammadex should be considered carefully. Future studies are warranted.

Hearing impaired patients have difficulty perceiving changes in their environment and communicating, which are relevant to anesthesia recovery. Effective communication between the patient and medical personnel during recovery is crucial. Administration of flumazenil after remimazolam allows rapid psychomotor recovery from sedation [14], even in patients with muscular 
dystrophy [15]. The administration of flumazenil after remimazolam anesthesia may be useful to assist in achieving good communication with hearing impaired patients.

Remimazolam, remifentanil, and rocuronium can be pharmacologically antagonized, which is a major advantage in their use. The rapid and clear response achieved with flumazenil in our patient may indicate the superiority of remimazolam over other sedatives. However, flumazenil may cause convulsions by antagonizing benzodiazepine effects [16-18], and should not be administered casually.

\section{Conclusions}

Administration of remimazolam, an ultrashort-acting benzodiazepine, and reversal with flumazenil enabled rapid and complete recovery from anesthesia in our patient with mitochondrial disease. Potential complications associated with flumazenil administration should be considered on an individual basis. Remimazolam is a feasible alternative to propofol and volatile anesthetics in patients with mitochondrial disease.

\section{Abbreviations}

TOF: Train-of-four

\section{Acknowledgements}

We thank Edanz (https://jp.edanz.com/ac) for editing a draft of this manuscript.

\section{Authors' contributions}

YS contributed to anesthetic management. YS, MD, and YN wrote the manuscript. The authors read and approved the final manuscript.

\section{Funding}

Financial support was not provided for this case report.

Availability of data and materials

Not applicable.

\section{Declarations}

Ethics approval and consent to participate

Not applicable.

\section{Consent for publication}

Written informed consent was obtained from the patient for publication of this case report.

\section{Competing interests}

The authors do not have any conflicts of interest regarding the content of this paper.

Received: 9 May 2021 Revised: 10 June 2021

Accepted: 15 June 2021 Published online: 23 June 2021

\section{References}

1. Niezgoda J, Morgan PG. Anesthetic considerations in patients with mitochondrial defects. Paediatr Anaesth. 2013;23(9):785-93. https://doi.org/1 0.1111/pan.12158

2. Footitt EJ, Sinha MD, Raiman JAJ, Dhawan A, Moganasundram S, Champion MP. Mitochondrial disorders and general anaesthesia: a case series and review. Br J Anaesth. 2008;100:436-41.
3. Yamamoto N, Okuyama H, Hiraumi H, Sakamoto T, Matsuura H, Ito J. The outcome of cochlear implantation for mitochondrial disease patients with syndromic hearing loss. Otol Neurotol. 2015;36(8):e129-33. https://doi.org/1 $0.1097 / \mathrm{MAO} .0000000000000817$.

4. Chow SY, Woon KL. General anesthesia for adults with mitochondrial myopathy. A A Case Rep. 2015;4(5):52-7. https://doi.org/10.1213/XAA. 0000000000000119

5. Kilpatrick GJ, McIntyre MS, Cox RF, Stafford JA, Pacofsky GJ, Lovell GG, et al. CNS 7056: a novel ultra-short-acting benzodiazepine. Anesthesiology. 2007; 107(1):60-6. https://doi.org/10.1097/01.anes.0000267503.85085.c0.

6. Gurnaney $H$, Brown A, Litman RS. Malignant hyperthermia and muscular dystrophies. Anesth Analg. 2009;109(4):1043-8. https://doi.org/10.1213/ane. ob013e3181aa5cf6.

7. Rafique MB, Cameron SD, Khan Q, Biliciler S, Zubair S. Anesthesia for children with mitochondrial disorders: a national survey and review. J Anesth. 2013;27(2):186-91. https://doi.org/10.1007/s00540-012-1488-1.

8. Kam PCA, Cardone D. Propofol infusion syndrome. Anaesthesia. 2007;62(7): 690-701. https://doi.org/10.1111/j.1365-2044.2007.05055.x.

9. Hsieh VC, Krane EJ, Morgan PG. Mitochondrial disease and anesthesia. Journal of Inborn Errors of Metabolism and Screening. 2017;5: 2326409817707770 .

10. Wisely NA, Cook PR. General anaesthesia in a man with mitochondrial myopathy undergoing eye surgery. Eur J Anaesthesiol. 2001;18(5):333-5. https://doi.org/10.1097/00003643-200105000-00009.

11. Finsterer J, Stratil U, Bittner R, Sporn P. Increased sensitivity to rocuronium and atracurium in mitochondrial myopathy. Can J Anaesth. 1998;45(8):7814. https://doi.org/10.1007/BF03012149.

12. Baumüller E, Schaller SJ, Chiquito Lama Y, Frick CG, Bauhofer T, Eikermann $M$, et al. Postoperative impairment of motor function at train-of-four ratio $\geq 0.9$ cannot be improved by sugammadex $\left(1 \mathrm{mg} \mathrm{kg}^{-1}\right)$. Br J Anaesth. 2015; 114:785-93.

13. Gurunathan U, Kunju SM, Stanton LML. Use of sugammadex in patients with neuromuscular disorders: a systematic review of case reports. BMC Anesthesiol. 2019;19(1):213. https://doi.org/10.1186/s12871-019-0887-3.

14. Chen X, Sang N, Song K, Zhong W, Wang H, Jiang J, et al. Psychomotor recovery following remimazolam-induced sedation and the effectiveness of flumazenil as an antidote. Clinical Therapeutics. 2020;42(4):614-24. https:// doi.org/10.1016/j.clinthera.2020.02.006

15. Morimoto Y, Yoshimatsu A, Yoshimura M. Anesthetic management for a patient with myotonic dystrophy with remimazolam. JA Clin Rep. 2021;7(1): 10. https://doi.org/10.1186/s40981-021-00413-3.

16. Schulze-Bonhage A, Elger CE. Induction of partial epileptic seizures by flumazenil. Epilepsia. 2000;41(2):186-92. https://doi.org/10.1111/j.1528-1157.2 000.tb00138.x.

17. Marchant B, Wray R, Leach A, Nama M. Flumazenil causing convulsions and ventricular tachycardia. BMJ. 1989;299(6703):860. https://doi.org/10.1136/ bmj.299.6703.860-b.

18. Penninga El, Graudal N, Ladekarl MB, Jürgens G. Adverse events associated with flumazenil treatment for the management of suspected benzodiazepine intoxication - a systematic review with meta-analyses of randomised trials. Basic \& Clinical Pharmacology \& Toxicology. 2016;1 18(1): 37-44. https://doi.org/10.1111/bcpt.12434.

\section{Publisher's Note}

Springer Nature remains neutral with regard to jurisdictional claims in published maps and institutional affiliations. 